\title{
MicroRNA-124 inhibits proliferation, invasion, migration and epithelial-mesenchymal transition of cervical carcinoma cells by targeting astrocyte-elevated gene-1
}

\author{
XIA ZHANG ${ }^{1}$, DAJUN CAI ${ }^{1}$, LIHUA MENG $^{2}$ and BING WANG ${ }^{1}$ \\ ${ }^{1}$ Department of Gynecology and Obstetrics, The Second Affiliated Hospital of Zhengzhou University, \\ Zhengzhou, Henan 450014; ${ }^{2}$ Department of Gynecology and Obstetrics, Qilu Hospital \\ of Shandong University, Jinan, Shandong 250012, P.R. China
}

Received March 13, 2016; Accepted August 1, 2016

DOI: $10.3892 /$ or.2016.5025

\begin{abstract}
MicroR NA-124 (miR-124) was reported to be attenuated in human cervical cancer (CC) specimens. However, its role in $\mathrm{CC}$ remains elusive. In the present study, quantitative real-time PCR (qRT-PCR) analysis demonstrated that miR-124 expression is significantly downregulated in human CC tissues and several CC cell lines. Transfection of miR-124 mimics in CC cell lines HeLa and SiHa markedly inhibits cell proliferative, migratory and invasive capacities, as well as the epithelial-mesenchymal transition (EMT) process in vitro. Further studies have identified astrocyte-elevated gene-1 (AEG-1) as a direct target gene of miR-124. miR-124 downregulated AEG-1 expression through interaction with its 3'-untranslated regions (3'-UTRs), and miR-124 expression was inversely correlated with AEG-1 levels in CC specimens. Moreover, exogenous overexpression of AEG-1 significantly rescued the miR-124-induced inhibition of cell proliferation, migration and invasion, as well as the EMT process in HeLa cells. In conclusion, these findings suggested that miR-124 was able to suppress cell proliferation, invasion and migration, as well as the EMT process in cervical carcinomas through
\end{abstract}

Correspondence to: Dr Xia Zhang, Department of Gynecology and Obstetrics, The Second Affiliated Hospital of Zhengzhou University, 2 Jingba Road, Jinshui District, Zhengzhou, Henan 450014, P.R. China E-mail: xiazhanggo@163.com

Abbreviations: CC, cervical cancer; miR-124, microRNA-124; EMT, epithelial-mesenchymal transition; AEG-1, astrocyte-elevated gene-1; MTDH, metadherin; LYRIC, lysine-rich CEACAM-1-associated protein; 3'-UTR, 3'-untranslated region; MMP-9, matrix metalloproteinase-9; AKR1C2, aldo-keto reductase family 1 member $\mathrm{C} 2$; NF1, neurofibromin 1

Key words: microRNA-124, cervical carcinoma, astrocyte-elevated gene-1, proliferation, migration, invasion, epithelial-mesenchymal transition directly targeting AEG-1. miR-124 and AEG-1 may be potential therapeutic targets for the treatment of cervical carcinoma.

\section{Introduction}

Cervical cancer (CC) is one of the most frequent gynecologic malignancies, accounting for significant morbidity and mortality in females worldwide (1). Tumor invasion and metastasis remain formidable obstacles to the effective treatment of this disease. Thus, research on the molecular mechanisms leading to invasion and metastatic dissemination of carcinoma cells are urgently needed for a more effective therapeutic strategy.

Astrocyte-elevated gene-1 (AEG-1), also known as metadherin (MTDH) and lysine-rich CEACAM1 co-isolate protein (LYRIC), is considered an important oncogene, whose expression is involved in carcinogenesis, tumor progression and chemotherapeutic resistance in several malignancies (2). Expression of AEG-1 is elevated in glioblastoma multiforme and contributes to tumor cell survival, proliferation and invasion (3). Overexpression of AEG-1 contributes to the neoplastic phenotype of bladder cancer cells by promoting survival, clonogenicity and migration (4). It is reported that AEG-1 is highly expressed in CC and associates with progression of cervical intraepithelial neoplasia and unfavorable prognosis $(5,6)$. This suggests that AEG-1 plays a crucial role in the progression of CC. However, the mechanism of upregulation of AEG-1 in cervical cancer has not yet been fully clarified. MicroRNAs (miRNAs or miR) are endogenous non-coding small RNAs, many of which are abnormally expressed in various types of cancers and involved in tumorigenesis and tumor development. miR-124 is one of the most studied and best characterized miRNAs with pivotal roles in several malignant processes, including tumor proliferation, motility and angiogenesis (7). It has been reported that miR-124 promoter is heavily methylated in CC cell lines, thus contributing to its slight expression in human $\mathrm{CC}$ specimens $(8,9)$. miR-124 was discovered to be involved in MALAT1-miR-124-RBG2 axis where it affects growth and invasion of high-risk human papillomavirus-positive CC cells (10). However, molecular mechanisms by which miR-124 achieve its effects in CC are still being elucidated. 
Recent studies revealed that several miRNAs may serve as potential regulatory mechanisms of AEG-1 in human cancers, such as miR-26a (11), miR-137 (12) and miR-375 (13). Intriguingly, bioinformatic analysis showed that AEG-1 harbored a potential miR-124 binding site in its 3'-untranslated regions ( 3 '-UTRs), suggesting that AEG-1 is a putative target of miR-124. In the present study, the expression of miR-124 was found to be inversely downregulated with the expression of AEG-1 in cervical carcinoma. Furthermore, it was shown that miR-124 can suppress CC cell proliferation, migration and invasion, as well as inhibit epithelial-mesenchymal transition (EMT) in cervical carcinoma through directly targeting AEG-1, suggesting that miR-124/AEG-1 axis has a pivotal role in the progression of $\mathrm{CC}$.

\section{Materials and methods}

Ethics statements. The present study was conducted with the approval from the Ethics Committee of the Second Affiliated Hospital of Zhengzhou University. Written informed consent was obtained from all patients before study initiation.

Tissue specimens. Pair-matched tumor and adjacent normal cervical tissues from $18 \mathrm{CC}$ patients were obtained from the Second Affiliated Hospital of Zhengzhou University, China. None of the patients had suffered from any other severe diseases that may affect the study. None of them received radiotherapy or chemotherapy before the tissues were obtained. All specimens were obtained during routine resection surgery, then immediately snap-frozen in liquid nitrogen, and stored at $-80^{\circ} \mathrm{C}$ for further analysis.

Cell culture. The human CC cell lines (HeLa, SiHa, CaSKi and C33A) and normal cervical epithelial cells (End1/E6E7) were purchased from the Chinese Academy of Sciences Cell Bank (Shanghai, China). HeLa, SiHa and C33A cells were cultured in Dulbecco's modified Eagle's medium (DMEM), CaSKi cells were cultured in RPMI-1640 medium (both from Gibco, Grand Island, NY, USA) supplemented with $10 \%$ heat-inactivated fetal bovine serum (FBS; Sigma-Aldrich, St. Louis, MO, USA), $100 \mathrm{U} / \mathrm{ml}$ penicillin and $100 \mathrm{U} / \mathrm{ml}$ streptomycin (Invitrogen, Carlsbad, CA, USA). End1/E6E7 cells were cultured in KER-SFM medium (Gibco). All cells were fostered in a humidified atmosphere chamber containing 5\% $\mathrm{CO}_{2}$ at $37^{\circ} \mathrm{C}$.

Cell transfection. HeLa and $\mathrm{SiHa}$ cells were respectively seeded into 24 -well plates $\left(1 \times 10^{6}\right.$ cells/well $)$ and grown to $70 \%$ confluency before transfection. miR-124 mimics (5'-GGCAUUCACCUCGUGCCUUA-3') and negative control mimics (NC; 5'-ACUACUGAGUGACAGUAGA-3') were synthesized by GenePharma (Shanghai, China) and transfected into prepared cells at a final concentration of $50 \mathrm{nM}$. For AEG-1 overexpression, the cDNA (without the 3'-UTR) of AEG-1 was cloned into pcDNA3.1 (+) (Invitrogen) to generate an AEG-1 overexpression vector. Cell transfections were performed with Lipofectamine ${ }^{\mathrm{TM}} 2000$ (Invitrogen) according to the manufacturer's instructions. Total RNA and protein contents were prepared $24 \mathrm{~h}$ post-transfection for further analysis.
Quantitative real-time PCR analysis. Total RNA was isolated from tissues or cultured cells using TRIzol reagent and reverse transcribed by M-MLV reverse transcriptase kit (both from Invitrogen) according to the manufacturer's protocols. Quantitative real-time PCR (qRT-PCR) was performed on an ABI 7900HT Fast Real-Time PCR system (Applied Biosystems, Foster City, CA, USA). All PCRs were conducted in triplicate and normalized to the internal control (GAPDH or U6). The relative expression levels were evaluated using the $2^{-\Delta \Delta \mathrm{Cq}}$ method. The specific primers were designed as follows: miR-124 RT primer, 5'-CTCAACTGGTGTCGTGGAGTCG GCAATTCAGTTGAGAGGCATTC-3'; miR-124 PCR primers forward, 5'-ACACTCCAGCTGGGTAAGGCACG CGGTGA-3' and reverse, 5'-TGGTGTCGTGGAGTCG-3'; U6 RT primer, 5'-TGGTGTCGTGGAGTCG-3'; U6 PCR primers forward, 5'-CTCGCTTCGGCAGCACA-3' and reverse, 5'-AA CGCTTCACGAATTTGCGT-3'; AEG-1 (MTDH) PCR primers forward, 5'-TGTTGAAGTGGCTCAGGG-3' and reverse, 5'-CAGGAAATGATGCGGTTG-3'; GAPDH PCR primers forward, 5'-CCACCCATGGCAAATTCCATG GCA-3' and reverse, 5'-TCTAGACGGCAGGTCAGGTCC ACC-3'.

Cell proliferation assay. Transfected cells were seeded into 24-well plates $\left(3 \times 10^{3}\right.$ cells/well) and cultured at $37^{\circ} \mathrm{C}$. Cellular proliferation was evaluated at the indicated time $(24,48,72$ or 96 h) using a Cell Counting Kit-8 (CCK-8; Sigma, St. Louis, MO, USA) in accordance with the manufacturer's protocol. After incubation with CCK- 8 solution for another $4 \mathrm{~h}$, the optical absorbance was measured at the wavelength of $450 \mathrm{~nm}$. The experiment was performed in triplicate.

Cell apoptosis assay. HeLa and $\mathrm{SiHa}$ cells were transiently transfected with miR-124 mimic or NC. Cells were harvested after $72 \mathrm{~h}$ by trypsinization. Double staining with FITC-Annexin V and propidium iodide (PI) was carried out using the FITC-Annexin $\mathrm{V}$ apoptosis detection kit (BD Biosciences, Bedford, MA, USA). Cells were analyzed by flow cytometry.

Cell invasion and migration assays. Cell invasion and migration abilities were determined using an $8-\mu \mathrm{m}$-pore polycarbonate membrane Boyden chamber insert in a Transwell apparatus (Costar, Cambridge, MA, USA), with and without Matrigel (BD Biosciences), respectively. Cells were harvested and resuspended in serum-free medium after $48 \mathrm{~h}$ transfection. For the invasion assays, $1 \times 10^{5}$ cells were prepared in $200 \mu \mathrm{l}$ of serum-free RPMI-1640 medium then placed in the upper chamber coated with Matrigel. For the migration assays, $3 \times 10^{4}$ cells were prepared in $200 \mu \mathrm{l}$ of serum-free RPMI-1640 medium and seeded into the upper chamber of the Transwell. The lower chamber was filled with RPMI-1640 medium containing $10 \% \mathrm{FBS}$ to induce chemotaxis. After incubation for $24 \mathrm{~h}$ at $37^{\circ} \mathrm{C}$ in a $5 \% \mathrm{CO}_{2}$ incubator, the invaded or migrated cells were fixed with $100 \%$ methanol, stained in $0.5 \%$ crystal violet (Sigma), and counted under a microscope. The results were averaged over three independent experiments.

Xenograft tumor model. To evaluate in vivo tumorigenesis, a CC xenograf mouse model was used. Female BALB/c 
athymic nude mice (4-6-weeks old) were purchased from the Laboratory Animal Center of Chinese Academy of Sciences (Shanghai, China). All animal experiments were performed with the guide and approval of the Institutional Animal Care and Use Committee of the Second Affiliated Hospital of Zhengzhou University. HeLa cells $\left(2 \times 10^{6}\right)$ stably transfected with miR-124 mimic or NC were injected subcutaneously into the flanks of nude mice $(n=8)$. Once palpable tumors developed, tumor volume was measured and calculated using the formula: Volume $=0.5 \mathrm{x}$ length $\mathrm{x}$ width $^{2}$. Thirty days after inoculation, the mice were euthanized and tumor weights were assessed.

Western blot analysis. Total proteins were extracted from CC samples or cells using RIPA buffer (Abcam, Cambridge, MA, USA). Protein concentrations were determined by BCA protein assay kit (Takara Bio Inc., Otsu, Japan). Twenty micrograms of protein was fractionated by $10 \%$ SDS-PAGE. After electrophoresis, proteins were transferred to nitrocellulose membranes (Millipore, Bedford, MA, USA). The blots were probed with the corresponding primary antibodies specific for either AEG-1, E-cadherin, $\beta$-catenin, N-cadherin, vimentin, twist, matrix metalloproteinase-9 (MMP-9), aldo-keto reductase family 1 member C2 (AKR1C2), neurofibromin 1 (NF1) or GAPDH (Abcam). Thereafter, the membranes were incubated with horseradish peroxidase (HRP)-conjugated goat anti-rabbit IgG or HRP-conjugated goat anti-mouse IgG (Abcam). Signals were visualized with an enhanced chemiluminescence system (Amersham Pharmacia Biotech, San Francisco, CA, USA), and analyzed using ImageJ software. Protein levels were determined by normalization to GAPDH.

Plasmid construction and luciferase assays. The wildtype 3'-UTR segment of AEG-1 was cloned downstream of the luciferase coding region in the psi-CHECK ${ }^{\mathrm{TM}}$ Vectors (Promega, Madison, WI, USA) to generate luciferase reporter vector AEG-1 3'-UTR-wt. The corresponding mutant constructs (AEG-1 3'-UTR-mut) were created by mutating the seed regions of the miR-124-binding sites. Cells were plated into 24-well plates until $80 \%$ confluence before transfection. The wild-type or mutant AEG-1 3'-UTR plasmid was transiently co-transfected with miR-124 mimics or NC into HeLa cells. Cell lysates were harvested $24 \mathrm{~h}$ post-transfection and luciferase activities were measured by the Dual-Luciferase Reporter Assay System (Promega). Luciferase activity was normalized by Renilla luciferase activity.

Statistical analysis. All statistical calculations were performed using SPSS software (version 19.0). Data were obtained from three repeated experiments and expressed as mean \pm standard deviation (SD). Differences between groups were subjected to the Student's-t test. Statistical significance was set at $\mathrm{P}<0.05$.

\section{Results}

Downregulation of miR-124 in CC tissues and cell lines. Expression of miR-124 in CC tissues was determined and compared to that in the adjacent normal tissues. It was shown
Table I. Association between miR-124 expression and clinicopathological parameters in study patients.

\begin{tabular}{|c|c|c|c|c|}
\hline \multirow[b]{2}{*}{ Clinical parameters } & \multirow[b]{2}{*}{ Cases } & \multicolumn{2}{|c|}{$\begin{array}{c}\text { Expression of } \\
\text { miR-124 }\end{array}$} & \multirow[b]{2}{*}{ P-value } \\
\hline & & $\begin{array}{l}\text { High } \\
(n=10)\end{array}$ & $\begin{array}{l}\text { Low } \\
(\mathrm{n}=8)\end{array}$ & \\
\hline Age (years) & $(27-61)$ & & & 0.704 \\
\hline$<45$ & 9 & 4 & 5 & \\
\hline$\geq 45$ & 9 & 6 & 3 & \\
\hline Tumor size $(\mathrm{cm})$ & & & & 0.683 \\
\hline$<3$ & 8 & 4 & 4 & \\
\hline$\geq 3$ & 10 & 6 & 4 & \\
\hline HPV infection & & & & 0.537 \\
\hline Positive & 10 & 6 & 4 & \\
\hline Negative & 8 & 4 & 4 & \\
\hline Pathological type & & & & 0.725 \\
\hline Squamous carcinoma & 12 & 7 & 5 & \\
\hline Adenocarcinoma & 6 & 3 & 3 & \\
\hline Tumor differentiation & & & & 0.364 \\
\hline Well/moderate & 9 & 6 & 3 & \\
\hline Poor & 9 & 4 & 5 & \\
\hline FIGO stage & & & & $0.042^{\mathrm{a}}$ \\
\hline $\mathrm{I}+\mathrm{II}$ & 11 & 5 & 6 & \\
\hline III+IV & 7 & 5 & 2 & \\
\hline Vascular invasion & & & & $0.034^{\mathrm{a}}$ \\
\hline Yes & 10 & 6 & 4 & \\
\hline No & 8 & 4 & 4 & \\
\hline Lymph node metastasis & & & & $0.027^{\mathrm{a}}$ \\
\hline Yes & 9 & 5 & 4 & \\
\hline No & 9 & 5 & 4 & \\
\hline AEG-1 expression & & & & $0.018^{\mathrm{a}}$ \\
\hline High & 11 & 4 & 7 & \\
\hline Low & 7 & 6 & 1 & \\
\hline
\end{tabular}

When the expression level was higher than or equal to the average, it was defined as high expression; ${ }^{\mathrm{P}} \mathrm{P}<0.05$. HPV, human papilloma virus; FIGO, International Federation of Gynecology and Obstetrics; AEG-1, astrocyte-elevated gene-1.

that miR-124 expression levels were significantly reduced in $\mathrm{CC}$ tissues (Fig. 1A; $\mathrm{P}<0.05$ ). We further evaluated the expression of miR-124 in CC cell lines. The results showed that the expression levels of miR-124 in all four CC cell lines (HeLa, SiHa, CaSKi and C33A) were much lower than that in End1/E6E7, a normal cervical epithelial cell line (Fig. 1B; $\mathrm{P}<0.05)$. We next tested the association between miR-124 and clinicopathological characteristics of CC patients (Table I). Consequently, low expression of miR-124 correlated with advanced International Federation of Gynecology and Obstetrics (FIGO) stage, vascular invasion and lymph node metastasis. 

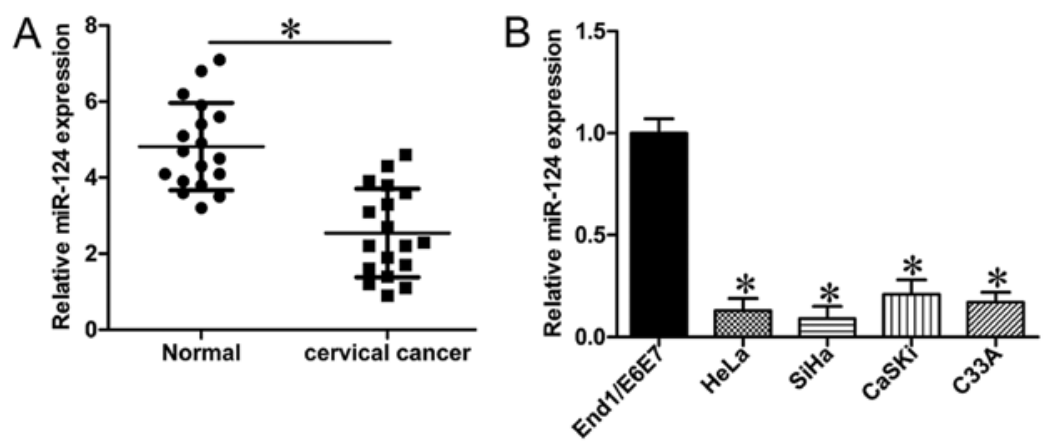

Figure 1. miR-124 expression is downregulated in cervical cancer. (A) Expression of miR-124 in each sample was quantitatively analyzed using RT-PCR and normalized with U6 mRNA; * $\mathrm{P}<0.05$, vs. normal tissues (normal). (B) Relative miR-124 expression levels in four cervical cancer lines (HeLa, SiHa, CaSKi and C33A) and a normal cervical epithelial cell line (End1/E6E7) were determined with RT-PCR; " $\mathrm{P}<0.05$, vs. End1/E6E7.
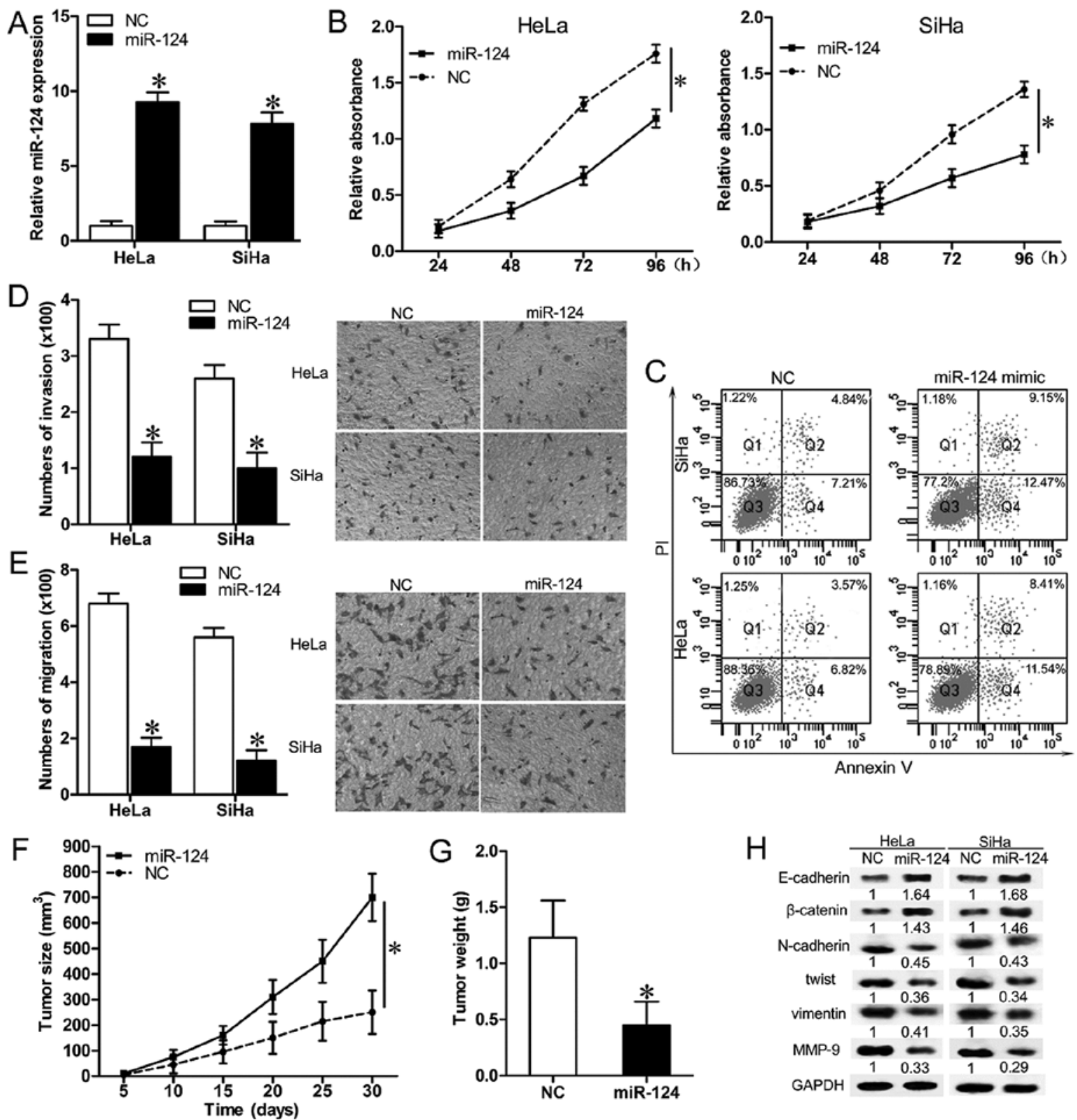

Figure 2. Overexpression of miR-124 suppresses cervical cancer progression. HeLa and SiHa cells were transiently transfected with miR-124-mimic (miR-124) or negative control mimic (NC). (A) Overexpression of miR-124 in HeLa and SiHa cells was validated using RT-PCR. (B) Cell growth curve of HeLa and $\mathrm{SiHa}$ cells was determined by CCK-8 assay. (C) Changes of apoptosis in HeLa and SiHa cells was determined by flow cytometric assay. (D and E) Migration and invasion abilities of HeLa and SiHa cells were measured using Transwell migration assay and Transwell invasion assay, respectively. (F) and (G) In vivo tumorigenesis assay was used to measure tumor volume (F) and tumor weight (G). (H) Expression of EMT markers and MMP-9 in HeLa and SiHa cells were evaluated by western blotting; ${ }^{*} \mathrm{P}<0.05$, vs. NC. 
A

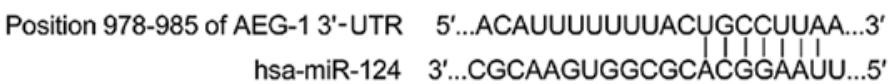
AEG-1 3 '-UTR-mut $\quad 5^{\prime}$...ACAUUUUUUUACUCCGUUCA....3'
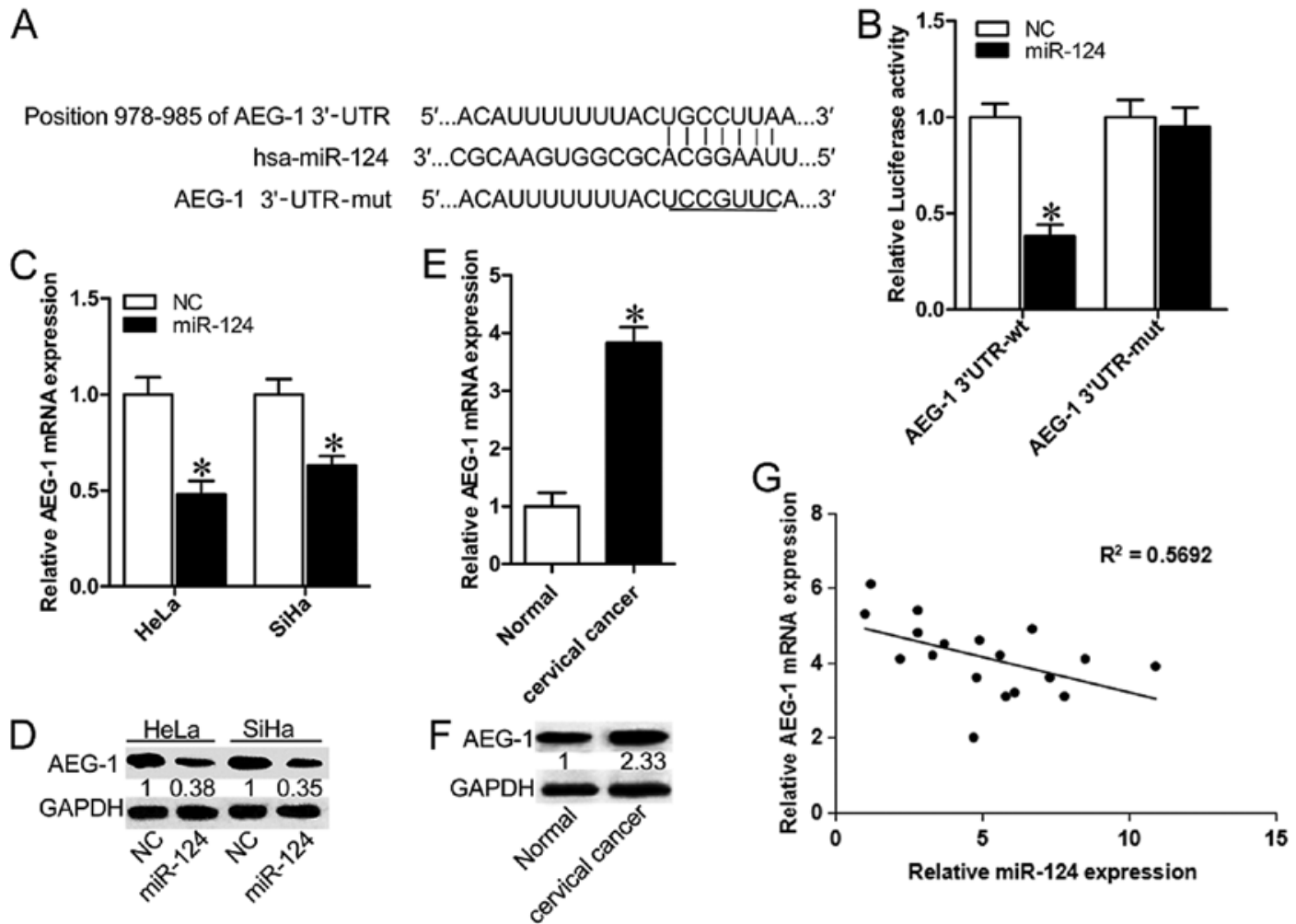

Figure 3. AEG-1 is a functional target for miR-124 in cervical cancer. (A) miR-124 and its putative binding sequences in the 3'-UTR of AEG-1. Mutations were generated in the complementary site that binds to the seed region of miR-124. (B) Luciferase reporter assay of the interaction between the 3'-UTR of AEG-1 and miR-124 was validated in HeLa cells. (C and D) AEG-1 mRNA and protein expression in HeLa and SiHa cells transfected with miR-124-mimic (miR-124) or negative control mimic (NC) were detected by quantitative RT-PCR and western blot assay, respectively. (E and F) Quantitative RT-PCR and western blot analysis revealed significantly higher AEG-1 mRNA and protein expression in human CC specimens compared with normal tissues (normal). (G) Spearman's correlation analysis showed that miR-124 expression was inversely correlated with AEG-1 mRNA expression in CC specimens; ${ }^{*} \mathrm{P}<0.05$, vs. NC or normal.

These results showed that the expression of miR-124 was downregulated in human CC tissues and cells, and miR-124 may be involved in the progression of cervical carcinoma.

Exogenous overexpression of miR-124 suppresses the proliferation, migration and invasion of $C C$ cells. To investigate the role of miR-124 in cervical carcinogenesis, miR-124 mimics or NC were transfected into HeLa and SiHa cells (Fig. 2A), and the biologic behavior of the cells were subsequently characterized. Cell growth curves indicated that upregulation of miR-124 significantly suppressed cell proliferation (Fig. 2B; $\mathrm{P}<0.05)$. Transfection of miR-124 leads to induction of apoptosis in both HeLa and SiHa cells (Fig. 2C). Overexpression of miR-124 significantly decreased the migratory and invasive capacities of HeLa and SiHa cells (Fig. 2D and E; P<0.05). Xenografted tumors in mice inoculated with miR-217 mimic infected HeLa cells grew much more slowly than those in mice inoculated with $\mathrm{NC}$ (Fig. $2 \mathrm{~F}$ and $\mathrm{G} ; \mathrm{P}<0.05$ ). These results confirm that miR-124 acts as a tumor-suppressor, and its upregulation inhibits cell proliferation, migration and invasion in $\mathrm{CC}$ cell lines.

Exogenous overexpression of miR-124 inhibits EMT of CC cells. As epithelial-mesenchymal transition (EMT) is thought to be critical for cancer cell invasion and metastasis, we next examined the expression of several EMT-related molecules (lower E-cadherin and $\beta$-catenin, higher $\mathrm{N}$-cadherin, twist and vimentin), and metastasis-related molecules (higher MMP-9) to determine whether miR-124 is involved in regulation of EMT in cervical carcinomas. In both HeLa and the SiHa cells, miR-124 overexpression increased the expression level of E-cadherin and $\beta$-catenin. Simultaneously, protein levels of $\mathrm{N}$-cadherin, twist, vimentin and MMP-9 showed robust downregulation in both HeLa and SiHa cells (Fig. 2H). These results indicate that exogenous overexpression of miR-124 in $\mathrm{CC}$ cells partially represses EMT in cervical carcinomas.

miR-124 targets AEG-1 3'-UTR and downregulates its expression in cervical carcinomas. Bioinformatics analysis using miRTarBase (http://mirtarbase.mbc.nctu.edu.tw/index.php) and TargetScan 7.0 (http://www.targetscan.org/vert_70/) identified that AEG-1 harbors a potential miR-124 binding site (Fig. 3A). To verify whether AEG-1 is the direct target of miR-124, luciferase report vectors that contain the putative miR-124 binding sites within AEG-1 3'-UTR were constructed. The overexpression of miR-124 significantly suppressed the luciferase activity of AEG-1 containing a wild-type 3'-UTR, but did not suppress activity of AEG-1 with a mutant 3'-UTR (Fig. 3B; P<0.05). Transfection of miR-124 mimic led to a significant decrease of AEG-1 mRNA and protein expression in HeLa and SiHa cells (Fig. 3C and D; $\mathrm{P}<0.05$ ). To further determine whether miR-124-mediated repression of AEG-1 is of clinical relevance, we measured the expression of miR-124 and AEG-1 in CC specimens and pair-matched normal tissues. 

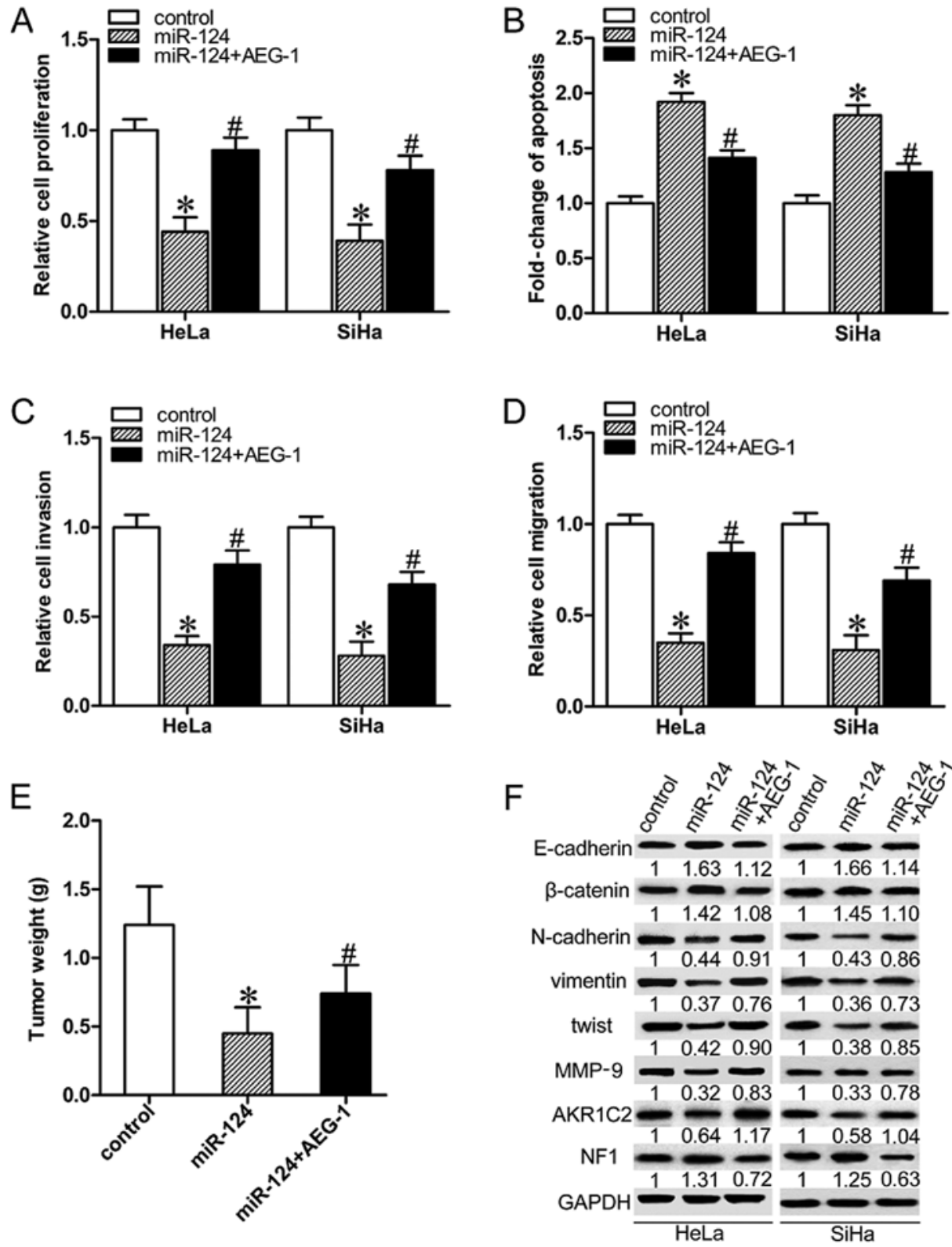

Figure 4. Overexpression of AEG-1 reverses the inhibitory effects of miR-124 in HeLa cells. HeLa cells (control) were transfected with miR-124-mimic (miR-124) alone, or co-transfected with AEG-1 overexpression vector (AEG-1). The cells were harvested for further testing $24 \mathrm{~h}$ after transfection. (A) Relative viabilities of HeLa and SiHa cells were determined by CCK-8 assay. (B) Changes of apoptosis in HeLa and SiHa cells were determined by flow cytometric assay. (C and D) Transwell invasion and migration analysis revealed that overexpression of AEG-1 significantly increased migratory and invasive ability of miR-124 overexpressing cells. (E) Changes of tumor weights after different transfection. (F) Western blot analysis revealed that overexpression of AEG-1 significantly increased the levels of $\mathrm{N}$-cadherin, twist, vimentin and MMP-9, and decreased E-cadherin and $\beta$-catenin expression in miR-124 overexpressing cells; ${ }^{\mathrm{P}} \mathrm{P}<0.05$, vs. control; ${ }^{\prime} \mathrm{P}<0.05$, vs. miR-124.

In contrast to the significantly lower expression of miR-124, AEG-1 showed higher levels in CC specimens when compared with the normal group (Fig. 3E and F; P $<0.05$ ). Furthermore, a negative correlation between mRNA levels of AEG-1 miR-124 and miR-124 was revealed by Spearman's correlation analysis (Fig. 3G). Together, these results suggest that AEG-1 is a target of miR-124 in cervical carcinomas.

Exogenous overexpression of AEG-1 partially reverses the inhibitory effects of miR-124 on CC cells. In order to investigate the contribution of AEG-1 to migration and invasion of human CC cells, AEG-1 was force-expressed along with miR-124 in HeLa and SiHa cells. Overexpression of AEG-1 partially nullified miR-124-mediated suppression of the proliferation, migration and invasion, and reduced miR-124-induced apoptosis in both HeLa and SiHa cells (Fig. 4A-D; P<0.05). The forced expression of AEG-1 increased the average weight of the miR-124 overexpressing tumors (Fig. 3E; P<0.05). Overexpression of AEG-1 partially restored miR-124-induced downregulation of N-cadherin, twist, vimentin and MMP-9, inhibited miR-124-induced upregulation of E-cadherin, $\beta$-catenin in HeLa and SiHa cells (Fig. 4F). The tumor mass was harvested and weighed. We next determined the expression levels of two novel AEG-1 downstream factors AKR1C2 and NF1. As shown in Fig. 4F, miR-124 decreased the expression of AKR1C2, but increased the expression of NF1. 
Collectively, our results demonstrated that miR-124 exerts suppressive effects on the progression of cervical carcinomas partly by targeting AEG-1.

\section{Discussion}

Numerous microRNAs (miRNAs) are known to play a key role in tumorigenesis due to their ability to act as either oncogenes or tumor suppressor genes. Various miRNAs, such as miR-34a, are considered to be ideal targets for therapeutic intervention in certain types of cancers (14). In the present study, we focused on miR-124, which is abnormally expressed in a variety of cancer types $(7,15,16)$. To date, evidence shows that miR-124 gene polymorphism is closely related to susceptibility to cervical cancer (CC) (17). In the present study, qRT-PCR analysis demonstrated that miR-124 was significantly downregulated in CC tissue samples and cancer cell lines. This finding was also verified by a study suggesting that abnormal promoter methylation and downregulation of miR-124 is a common event during cervical carcinogenesis (9).

Recently, reports also indicated multiple functions of miRNAs in cervical carcinomas. For example, miR-506 acts as a tumor suppressor in cervical carcinomas (18). The present study revealed that exogenous overexpression of miR-124 was able to markedly suppress cell proliferation and induce cell apoptosis in two CC cell lines. The introduction of miR-124 in HeLa and SiHa cells resulted in markedly decreased migratory and invasive behavior, consistent with previous studies showing that miR-124 inhibited the migration and invasion of ovarian cancer cells (19), and suppressed tumor growth and metastasis in nasopharyngeal carcinoma (20). Moreover, miR-124 overexpressed in CC cells exhibited an increasing E-cadherin and $\beta$-catenin expression, and decreasing $\mathrm{N}$-cadherin, twist, vimentin and MMP-9 expression, indicating that miR-124 inhibits EMT and metastasis in CC cases. The above observations suggest that miR-124 acts as a tumor-suppressor in CC, and ectopic expression of miR-124 may be helpful for treatment of cervical carcinoma. However, the specific regulatory mechanism and signal transduction network are still poorly understood and need to be further explored.

Knockdown of AEG-1 inhibits migration and invasion in colon cancer, suppresses metastasis in liver cancer (21), and decreases protective autophagy and chemosensitization of cancer cells (22). Several miRNAs, such as miR-145 and miR-217, were found to play an important role in tumor occurrence and development by regulating the expression level of AEG-1 in human cancers $(23,24)$. The present study revealed, for the first time, the involvement of miR-124 in tumorigenesis through targeting AEG-1 in cervical carcinoma. miR-124 downregulated AEG-1 expression through interaction with its 3'-UTR, and overexpression of miR-124 significantly suppressed AEG-1 expression in vitro. Accumulating studies have reported that AEG-1 is highly expressed in CC and is associated with cervical carcinoma progression and angiogenesis $(5,25)$. In the present study, higher expression of AEG-1 was observed, and was inversely correlated with miR-124 levels in CC specimens. Furthermore, exogenous overexpression of AEG-1 reversed the inhibitory effects of miR-124 on EMT and metastasis in CC cells. In accordance with the effect of AEG-1 silencing (21), overexpression of miR-124 also results in downregulation of AKR1C2 and upregulation of NF1. Both AKR1C2 and NF1 were involved in the process of proliferation and migration in vitro and in vivo $(26,27)$. Thus, the abnormal expression of AEG-1/AKR1C2/NF1 may contribute to the molecular mechanisms of inhibition of miR-124 on cell proliferation and migration in CC.

However, the present study did not clarify the specific mechanism by which AEG-1 affects EMT in CC. Both miR-124 and AEG-1 are involved in the EMT process though various signaling networks and effector targets, such as PI3K/Akt, NF- $\kappa B, W n t / \beta$-catenin and TGF- $\beta$ pathways $(28,29)$. According to existing research, PI3K/Akt and Wnt/ $\beta$-catenin seems to be more critical in the EMT process in CC. A previous study showed that AEG-1 mediates CCL20/CCR6-induced EMT development via both ERK1/2 and Akt signaling pathways in CC (30). Another study demonstrated that AEG-1 promotes EMT via the Wnt signaling pathway in cervical squamous cell carcinoma (31). Previously it was suggested that overexpression of miR-124 significantly suppressed migration and invasion and the activity of non-canonical Wnt signaling in osteosarcoma cells (32), while AEG-1 can activate Wnt signaling to promote metastasis in tongue squamous cell carcinoma. Thus, miR-124 mediated downregulation of AEG-1 may regulate the EMT process mainly via the Wnt signaling in CC. The specific mechanism in this process remains to be elucidated in the future.

Taken together, our results indicated that miR-124 is significantly downregulated in cervical carcinoma. miR-124 may suppress CC cell proliferation, migration and invasion, as well as inhibit EMT and metastasis in cervical carcinoma through directly targeting AEG-1. Our findings suggest that the miR-124/AEG-1 link has considerable value as a potential therapeutic target in cervical carcinoma cases.

\section{References}

1. Bhat S, Kabekkodu SP, Noronha A and Satyamoorthy K: Biological implications and therapeutic significance of DNA methylation regulated genes in cervical cancer. Biochimie 121: 298-311, 2016.

2. Shi $X$ and Wang $X$ : The role of MTDH/AEG-1 in the progression of cancer. Int J Clin Exp Med 8: 4795-4807, 2015.

3. Hu B, Emdad L, Bacolod MD, Kegelman TP, Shen XN, Alzubi MA, Das SK, Sarkar D and Fisher PB: Astrocyte elevated gene-1 interacts with Akt isoform 2 to control glioma growth, survival, and pathogenesis. Cancer Res 74: 7321-7332, 2014.

4. Nikpour M, Emadi-Baygi M, Fischer U, Niegisch G, Schulz WA and Nikpour P: MTDH/AEG-1 contributes to central features of the neoplastic phenotype in bladder cancer. Urol Oncol 32: 670-677, 2014.

5. Long M, Dong K, Gao P, Wang X, Liu L, Yang S, Lin F, Wei J and Zhang H: Overexpression of astrocyte-elevated gene-1 is associated with cervical carcinoma progression and angiogenesis. Oncol Rep 30: 1414-1422, 2013.

6. Huang K, Li LA, Meng Y, You Y, Fu X and Song L: High expression of astrocyte elevated gene-1 (AEG-1) is associated with progression of cervical intraepithelial neoplasia and unfavorable prognosis in cervical cancer. World J Surg Oncol 11: 297, 2013.

7. Wang X, Wu Q, Xu B, Wang P, Fan W, Cai Y, Gu X and Meng F: MiR-124 exerts tumor suppressive functions on the cell proliferation, motility and angiogenesis of bladder cancer by fine-tuning UHRF1. FEBS J 282: 4376-4388, 2015.

8. Wilting SM, van Boerdonk RA, Henken FE, Meijer CJ, Diosdado B, Meijer GA, le Sage C, Agami R, Snijders PJ and Steenbergen RD: Methylation-mediated silencing and tumour suppressive function of hsa-miR-124 in cervical cancer. Mol Cancer 9: 167, 2010. 
9. Jiménez-Wences H, Martínez-Carrillo DN, Peralta-Zaragoza O, Campos-Viguri GE, Hernández-Sotelo D, Jiménez-López MA, Muñoz-Camacho JG, Garzón-Barrientos VH, Illades-Aguiar B and Fernández-Tilapa G: Methylation and expression of miRNAs in precancerous lesions and cervical cancer with HPV16 infection. Oncol Rep 35: 2297-2305, 2016.

10. Liu S, Song L, Zeng S and Zhang L: MALAT1-miR-124-RBG2 axis is involved in growth and invasion of HR-HPV-positive cervical cancer cells. Tumour Biol 37: 633-640, 2016.

11. Liu P, Tang H, Chen B, He Z, Deng M, Wu M, Liu X, Yang L, Ye F and Xie X: miR-26a suppresses tumour proliferation and metastasis by targeting metadherin in triple negative breast cancer. Cancer Lett 357: 384-392, 2015.

12. Guo J, Xia B, Meng F and Lou G: miR-137 suppresses cell growth in ovarian cancer by targeting AEG-1. Biochem Biophys Res Commun 441: 357-363, 2013.

13. He XX, Chang Y, Meng FY, Wang MY, Xie QH, Tang F, Li PY, Song YH and Lin JS: MicroRNA-375 targets AEG-1 in hepatocellular carcinoma and suppresses liver cancer cell growth in vitro and in vivo. Oncogene 31: 3357-3369, 2012.

14. Di Martino MT, Leone E, Amodio N, Foresta U, Lionetti M, Pitari MR, Cantafio ME, Gullà A, Conforti $\mathrm{F}$, Morelli E, et al: Synthetic miR-34a mimics as a novel therapeutic agent for multiple myeloma: In vitro and in vivo evidence. Clin Cancer Res 18: 6260-6270, 2012.

15. Cai JJ, Qi ZX, Chen LC, Yao Y, Gong Y and Mao Y: miR-124 suppresses the migration and invasion of glioma cells in vitro via Capn4. Oncol Rep 35: 284-290, 2016.

16. Jiang L, Lin T, Xu C, Hu S, Pan Y and Jin R: miR-124 interacts with the Notch1 signalling pathway and has therapeutic potential against gastric cancer. J Cell Mol Med 20: 313-322, 2016.

17. Wu H and Zhang J: miR-124 rs531564 polymorphism influences genetic susceptibility to cervical cancer. Int J Clin Exp Med 7: 5847-5851, 2014

18. Wen SY, Lin Y, Yu YQ, Cao SJ, Zhang R, Yang XM, Li J, Zhang YL, Wang YH, Ma MZ, et al: miR-506 acts as a tumor suppressor by directly targeting the hedgehog pathway transcription factor Gli3 in human cervical cancer. Oncogene 34: 717-725, 2015.

19. Zhang H, Wang Q, Zhao Q and Di W: MiR-124 inhibits the migration and invasion of ovarian cancer cells by targeting SphK1. J Ovarian Res 6: 84, 2013.

20. Peng XH, Huang HR, Lu J, Liu X, Zhao FP, Zhang B, Lin SX, Wang L, Chen HH, Xu X, et al: MiR-124 suppresses tumor growth and metastasis by targeting Foxq1 in nasopharyngeal carcinoma. Mol Cancer 13: 186, 2014.
21. Li C, Wu X, Zhang W, Li J, Liu H, Hao M, Wang J, Zhang H, Yang G, Hao M, et al: AEG-1 promotes metastasis through downstream AKR1C2 and NF1 in liver cancer. Oncol Res 22: 203-211, 2014.

22. Huang Y and Li LP: Progress of cancer research on astrocyte elevated gene-1/Metadherin (Review). Oncol Lett 8: 493-501, 2014.

23. Wang M, Wang J, Deng J, Li X, Long W and Chang Y: MiR-145 acts as a metastasis suppressor by targeting metadherin in lung cancer. Med Oncol 32: 344, 2015.

24. Wang B, Shen ZL, Jiang KW, Zhao G, Wang CY, Yan YC, Yang Y, Zhang JZ, Shen C, Gao ZD, et al: MicroRNA-217 functions as a prognosis predictor and inhibits colorectal cancer cell proliferation and invasion via an AEG-1 dependent mechanism. BMC Cancer 15: 437, 2015.

25. Yu JQ, Zhou Q, Zhu H, Zheng FY and Chen ZW: Overexpression of astrocyte elevated gene-1 (AEG-1) in cervical cancer and its correlation with angiogenesis. Asian Pac J Cancer Prev 16: 2277-2281, 2015.

26. Ji Q, Chang L, Stanczyk FZ, Ookhtens M, Sherrod A and Stolz A: Impaired dihydrotestosterone catabolism in human prostate cancer: Critical role of AKR1C2 as a pre-receptor regulator of androgen receptor signaling. Cancer Res 67: 1361-1369, 2007.

27. Baek ST and Tallquist MD: Nf1 limits epicardial derivative expansion by regulating epithelial to mesenchymal transition and proliferation. Development 139: 2040-2049, 2012.

28. Zoni E, van der Pluijm G, Gray PC and Kruithof-de Julio M: Epithelial plasticity in cancer: Unmasking a microRNA network for TGF- $\beta$-, Notch-, and Wnt-mediated EMT. J Oncol 2015: 198967,2015

29. Lee SG, Kang DC, DeSalle R, Sarkar D and Fisher PB: AEG-1/MTDH/LYRIC, the beginning: Initial cloning, structure, expression profile, and regulation of expression. Adv Cancer Res 120: 1-38, 2013.

30. Zhang J, Zhu D, Lv Q, Yi Y, Li F and Zhang W: The key role of astrocyte elevated gene-1 in CCR6-induced EMT in cervical cancer. Tumour Biol 36: 9763-9767, 2015.

31. Song E, Yu W, Xiong X, Kuang X, Ai Y and Xiong X: Astrocyte elevated gene-1 promotes progression of cervical squamous cell carcinoma by inducing epithelial-mesenchymal transition via Wnt signaling. Int J Gynecol Cancer 25: 345-355, 2015.

32. Zhang C, Hu Y, Wan J and He H: MicroRNA-124 suppresses the migration and invasion of osteosarcoma cells via targeting ROR2-mediated non-canonical Wnt signaling. Oncol Rep 34: 2195-2201, 2015. 